\title{
An Update on Immune Checkpoint Inhibitor-related Hypophysitis
}

\author{
Michael J Mortensen, ${ }^{1}$ Oliver Oatman, ${ }^{2}$ Amir Azadi, ${ }^{3}$ Ekokobe Fonkem, ${ }^{3}$ and Kevin CJ Yuen ${ }^{4}$ \\ 1. University of Arizona College of Medicine, Phoenix, AZ, USA; 2. Division of Endocrinology, Barrow Neurological Institute at Phoenix Children's \\ Hospital, Phoenix, AZ, USA; 3. Department of Neurology and Neuro-Oncology, Barrow Neurological Institute/lvy Brain Center at Phoenix, St. Joseph's \\ Hospital and Medical Center, Phoenix, AZ, USA; 4. Department of Neuroendocrinology and Neurosurgery, Barrow Neurological Institute, University of \\ Arizona College of Medicine and Creighton School of Medicine, Phoenix, AZ, USA
}

DOI: https://doi.org/10.17925/USE.2020.16.2.117

mmune checkpoint inhibitors (ICIS) are a novel form of immunotherapy increasingly used in the treatment of various cancers. These agents are monoclonal antibodies that target specific glycoproteins (cytotoxic T-lymphocyte-associated protein 4 [CTLA-4], programmed cell death protein 1 [PD-1]) on T cells or ligands (programmed death-ligand 1 [PD-L1]) on antigen producing cells. Blockade of CTLA-4 and PD-1 (or its ligand PD-L1) by ICIs results in T-cell activation and tumor cell death. Initially used in adults, ICI use is now also expanding into the pediatric population. While ICIs have proven to be effective in the treatment of certain cancers, they also cause immune-mediated endocrinopathies. Immune-mediated hypophysitis is often unrecognized and can range from isolated pituitary hormone deficiency to, rarely, panhypopituitarism. Severe cases can cause pituitary gland enlargement that can result in visual disturbances due to compression on the optic chiasm, and potentially death from adrenal crisis. Treatment of hypophysitis depends on the severity of illness and ranges from initiation of hormone replacement, discontinuation of ICls, and administration of high glucocorticoid doses if mass-effect symptoms are present. Clinicians must be aware of ICl-related hypophysitis in order to promptly recognize and effectively treat this condition. Screening for endocrine dysfunction should continue even after completion of immunotherapy. In this review, we provide an update on the mechanism, incidence, clinical manifestations, and recommendations on diagnosis and treatment of ICl-related hypophysitis, specifically focusing on the use and dosing regimens of glucocorticoids in different clinical scenarios.

\section{Keywords}

Immune checkpoint inhibitors, hypophysitis, cancer, pituitary gland, adult, children

Disclosure: Michael J Mortensen, Oliver Oatman, Amir Azadi, Ekokobe Fonkem and Kevin CJ Yuen have no financial or non-financial relationships or activities to declare in relation to this article.

Review Process: Double-blind peer review.

Compliance with Ethics: This article involves a review of the literature and does not report on new clinical data, or any studies with human or animal subjects performed by any of the authors.

Authorship: The named authors meet the Internationa Committee of Medical Journal Editors (ICMJE) criteria for authorship of this manuscript, take responsibility for the integrity of the work as a whole, and have given final approval for the version to be published.

Access: This article is freely accessible at touchENDOCRINOLOGY.com (c) Touch Medical Media 2020

Received: July 27, 2020

Accepted: October 16, 2020

Published Online: December 15, 2020

Citation: US Endocrinology. 2020;16(2):117-24

Corresponding Author: Kevin CJ Yuen, Barrow

Pituitary Center, Barrow Neurological Institute,

124 W. Thomas Rd, Suite 300, Phoenix, AZ 85013, USA.

E: kevin.yuen@dignityhealth.org

Support: No funding was received in

the publication of this article.
The use of immunotherapy in the treatment of cancer has been revolutionary, opening a new era in the fight against cancer. The principle of immunotherapy involves using pharmacotherapy to harness the power of the body's own immune system to destroy cancer cells. ${ }^{1}$ Immune checkpoint inhibitors (ICIS) are a novel form of pharmacologic immunotherapy used in the treatment of various cancers. ${ }^{2}$ Immune checkpoints refer to a plethora of inhibitory pathways hardwired into the immune system that are crucial for maintaining self-tolerance and modulating the duration and amplitude of physiological immune responses in peripheral tissues in order to minimize collateral tissue damage. ${ }^{3}$ These agents are a unique class of monoclonal antibodies that target specific cell surface proteins involved in immune system inhibition, and are collectively known as "checkpoint inhibitors". ${ }^{3}$ The net effect of checkpoint blockade is activation of the immune system against tumor cells. Initially described in 1996, ${ }^{4}$ ICls have been on the market since 2011, with seven agents currently approved by the Food and Drug Administration (FDA). They are utilized as monotherapy, as adjuvant therapy in combination with other IClS, and in combination with standard chemotherapy regimens. ${ }^{5,6}$ With proven efficacy in the treatment of several types of cancer, their use has increased substantially over the past decade, including expansion into the pediatric oncology world. Table 1 displays the main cancers which currently have approval from the FDA for treatment with ICIS, and the associated endocrinopathies associated with these agents.

\section{Mechanism of action of immune checkpoint inhibitors}

Immune system $\mathrm{T}$ cells provide essential protection to the body by identifying and eradicating potentially harmful pathogens. ${ }^{8}$ Antigen-presenting cells communicate with $\mathrm{T}$ cells via interaction with receptors on the T-cell surface. ${ }^{8}$ Certain receptors activate T cells, while others are inhibitory. ${ }^{8}$ Together, these counter-regulatory receptors serve as a metaphorical "gas and brake pedal" of the immune system, allowing the T cell to activate or quiesce in response to specific antigens. Two T-cell receptors, cytotoxic T-lymphocyte-associated protein $4(\mathrm{CTLA}-4)^{4}$ and programmed cell death protein 1 (PD-1) as well as its ligand (PD-L1), , are of particular importance, as these are the primary targets of ICI therapy. CTLA-4 and PD-1 are receptors capable of inhibiting T cells after an antigen encounter, resulting in a return to a resting state. ${ }^{10}$ 
Table 1: Indications and main associated endocrinopathies of immune checkpoint inhibitors currently approved by the US Food and Drug Administration

\begin{tabular}{|c|c|c|c|c|c|}
\hline Drug & Manufacturer & Target & Year of approval & Indication & Main endocrinopathies \\
\hline Ipilimumab (Yervoy $\left.{ }^{\circledR}\right)$ & Bristol Myers Squibb & CTLA-4 & 2011 & $\begin{array}{l}\text { Malignant melanoma } \\
\text { Renal cell carcinoma } \\
\text { Colorectal carcinoma } \\
\text { Hepatocellular carcinoma }\end{array}$ & Hypopituitarism \\
\hline Nivolumab (Opdivo $\left.{ }^{\circledast}\right)$ & Bristol Myers Squibb & PD-1 & 2014 & $\begin{array}{l}\text { Metastatic melanoma } \\
\text { Non-small cell lung cancer } \\
\text { Advanced renal cell carcinoma } \\
\text { Hodgkin lymphoma } \\
\text { Head and neck squamous cell carcinoma } \\
\text { Gastric carcinoma } \\
\text { Metastatic colorectal cancer } \\
\text { Malignant mesothelioma } \\
\text { Colorectal carcinoma } \\
\text { Esophageal carcinoma }\end{array}$ & $\begin{array}{l}\text { Hypothyroidism } \\
\text { Hyperthyroidism }\end{array}$ \\
\hline Pembrolizumab (Keytruda ${ }^{\circledR}$ ) & Merck & PD-1 & 2014 & $\begin{array}{l}\text { Malignant melanoma } \\
\text { Non-small cell lung carcinoma } \\
\text { Head and neck squamous cell carcinoma } \\
\text { Hodgkin lymphoma } \\
\text { Large B-cell lymphoma } \\
\text { Urothelial carcinoma } \\
\text { Microsatellite instability-high cancer }\end{array}$ & $\begin{array}{l}\text { Hypothyroidism } \\
\text { Hyperthyroidism }\end{array}$ \\
\hline Atezolizumab (Tecentriq ${ }^{\circledR}$ ) & Genentech & PD-L1 & 2016 & $\begin{array}{l}\text { Urothelial carcinoma } \\
\text { Metastatic non-small cell lung cancer } \\
\text { Hepatocellular carcinoma }\end{array}$ & Hypothyroidism \\
\hline Durvalumab (Imfinzi $\left.{ }^{\circledR}\right)$ & AstraZeneca & PD-L1 & 2017 & $\begin{array}{l}\text { Non-small cell lung cancer } \\
\text { Urothelial carcinoma }\end{array}$ & $\begin{array}{l}\text { Hypothyroidism } \\
\text { Hyperthyroidism }\end{array}$ \\
\hline Avelumab (Bavencio ${ }^{\circledR}$ ) & EMD Serono & PD-L1 & 2017 & $\begin{array}{l}\text { Merkel cell carcinoma } \\
\text { Renal cell carcinoma }\end{array}$ & Hypothyroidism \\
\hline Cemiplimab (Libtayo ${ }^{\circledR}$ ) & Regeneron & PD-1 & 2018 & Metastatic cutaneous squamous cell carcinoma & Hypothyroidism \\
\hline
\end{tabular}

CTLA-4 = cytotoxic T-lymphocyte-associated protein 4; PD-1 = programmed cell death protein 1; PD-L1 = programmed death-ligand 1.

CTLA-4 is a member of the immunoglobulin superfamily, and once bound to ligands, CD80 and CD86, results in inhibition of T-cell proliferation and interleukin-2 (IL-2) production. ${ }^{11}$ PD-1 is also a member of the immunoglobulin superfamily, but is more widely expressed than CTLA-4 and can be detected on activated T cells, B cells, and natural killer cells. ${ }^{12}$ PD-1 binds to PD-L1 to activate inhibitory signaling pathways to suppress the immune response. ${ }^{12,13}$ Ipilimumab (Yervoy ${ }^{\circledR}$, Bristol Myers Squibb, New York, NY, USA) is an anti-CTLA-4 monoclonal antibody, whereas pembrolizumab (Keytruda ${ }^{\circledR}$, Merck, Kenilworth, NJ, USA), nivolumab (Opdivo ${ }^{\circledR}$, Bristol Myers Squibb), and cemiplimab (Libtayo ${ }^{\circledR}$, Regeneron, Tarrytown, NY, USA) are anti-PD-1 antibodies. PD-L1 inhibitors are unique in that they target the ligand on antigen presenting cells rather than the T-cell receptor. There are currently three PD-L1 inhibitors available on the market: atezolizumab (Tecentriq ${ }^{\circledR}$, Genentech, South San Francisco, CA, USA), durvalumab (Imfinzi ${ }^{\oplus}$, AstraZeneca, Cambridge, UK), and avelumab (Bavencio ${ }^{\oplus}$, EMD Serono, Darmstadt, Germany). All of the listed medications are designed for intravenous administration and are dosed at variable schedules.

\section{Current uses of immune checkpoint inhibitors}

Ipilimumab was the first ICl to be approved by the FDA, in 2011, for treatment of malignant melanoma, after showing significant improvement in overall survival. ${ }^{14,15}$ Since then, the PD-1 inhibitors pembrolizumab and nivolumab have been approved in 2014 for the treatment of malignant melanoma, and their use has subsequently expanded to include non-small cell lung cancer, Hodgkin's lymphoma, head and neck squamous cell carcinoma, bladder cancer, and advanced gastric cancer.13,16-18 Combination therapy of ipilimumab plus nivolumab was approved in 2016 for malignant melanoma, based on the pivotal phase III CheckMate 067 trial, in which a sustained survival benefit was demonstrated..$^{19}$ In 2016, PD-L1 inhibitors gained approval with atezolizumab for advanced non-small cell lung cancer and urothelial (bladder) cancer, ${ }^{20}$ and in 2017, durvalumab for metastatic urothelial carcinoma, ${ }^{21}$ and avelumab for Merkel cell carcinoma and urothelial carcinoma. ${ }^{22}$ The newest addition is cemiplimab, a PD-1 inhibitor approved in 2018 for metastatic and locally advanced cutaneous squamous cell carcinoma. ${ }^{23}$ More recently, ICIs have been used as adjuvant therapy to standard chemotherapy regimens and are being evaluated for use as neoadjuvant agents as well. ${ }^{5,6}$

\section{Endocrine side effects of immune checkpoint inhibitors}

The intent of $\mathrm{ICl}$ therapy is to selectively activate the immune system against tumor cells. Unfortunately, this is not always the case, and immune destruction of normal host tissue is a well-documented side effect of $\mathrm{ICl}$ 
therapy? Immune-mediated side effects can be seen in both non-endocrine as well as endocrine organs. Non-endocrine side effects commonly affect the dermatologic, pulmonary, gastrointestinal, and central nervous systems. ${ }^{24-26}$ Endocrine organs primarily affected by $\mathrm{ICI}$ therapy include the thyroid, pituitary, adrenal, pancreas, gonads, and rarely, the parathyroid glands. ${ }^{52427-29}$ The incidence varies by organ and by the type of $\mathrm{ICl}$ and its dosing, with CTLA-4 inhibitors being more likely to cause hypophysitis, and PD-1 and PD-L1 inhibitors more likely to result in thyroid dysfunction (e.g., hypothyroidism, thyrotoxicosis, painless thyroiditis, or even "thyroid storm").7.30 Combination therapy, while may be more efficacious in terms of treatment for the cancer, unfortunately results in higher rates of immune-related adverse events (irAEs). ${ }^{31}$ Additionally, irAEs have been reported to occur after months or years from the final dose of ICI therapy.25

ICl-related hypophysitis is of particular concern given the number of hormonal axes that can be affected with the involvement of only one endocrine gland. Adrenocorticotropic hormone (ACTH) deficiency is most frequently observed (20-75\%), followed by luteinizing hormone (LH)/follicle-stimulating hormone (FSH) (15-60\%) and thyroid-stimulating hormone (TSH) (25-58\%) deficiencies, and less commonly, growth hormone (5-41\%) and prolactin (13-25\%) deficiencies; while multiple pituitary hormone axes can be affected with resulting panhypopituitarism (three or more pituitary hormone deficits) in up to $50 \%$ of cases. ${ }^{732}$ However, hyperprolactinemia and diabetes insipidus are rare, affecting only $9 \%$ and $1 \%$ of patients, respectively.3.34

The plausible mechanism/s for hyperprolactinemia associated with hypophysitis include disruption of the inhibitory hypothalamic dopaminergic signals due to pituitary stalk inflammation or compression, concomitant hypothalamic inflammation leading to decreased dopamine synthesis, or the presence of lactotroph-stimulating auto-antibodies causing prolactin synthesis and release. ${ }^{35}$ In contrast, metastases to the pituitary gland have a predilection for the posterior pituitary, and thus are much more likely to induce diabetes insipidus. ${ }^{36}$ The presence of diabetes insipidus, therefore, may help differentiate hypophysitis from metastatic disease.

Unlike other organs, where effects can often be successfully treated with ICI discontinuation and high-dose glucocorticoid therapy, damage to pituitary cells-particularly corticotrophs-is often permanent and potentially life-threatening if not detected. ${ }^{7}$ This notion was demonstrated in a recent prospective observational study by Kassi et al., ${ }^{32}$ where none of the 29 patients that developed ICI-related hypophysitis recovered at 15 months from ACTH deficiency, and six out of seven (86\%) and one out of seven (14\%) patients recovered from TSH and gonadotropic deficits, respectively. Notably, the incidence of hypophysitis is higher with use of anti-CTLA-4 inhibitors, such as ipilimumab (0-17\%), than with PD-1 inhibitors nivolumab and pembrolizumab $(0.5-2.0 \%)$, ${ }^{26}$ whereas PD-L1 inhibitors rarely cause hypophysitis. ${ }^{37,38}$ The incidence is increased with higher ipilimumab doses, and older age and male gender are risk factors. ${ }^{39-41}$

The mechanism by which CTLA-4 inhibitors disproportionally affect the pituitary gland may be explained by the presence of CTLA-4 within normal pituitary cells in certain individuals. ${ }^{42} \mathrm{~A}$ study by Caturegli et al., examining CTLA-4 expression within the pituitary gland in six patients treated with CTLA-4 inhibitors (one of which had clinical as well as pathologic hypophysitis), demonstrated variable intracytoplasmic expression of CTLA-4, with the highest expression being in patients with hypophysitis. ${ }^{43}$
These authors postulated that CTLA-4 inhibitors may bind to normal pituitary cells in which CTLA-4 granules are present, thereby causing type II as well as type IV hypersensitivity reactions, ultimately resulting in complement activation and macrophage/autoreactive T lymphocyte infiltration. ${ }^{43}$ However, the true purpose of intracytoplasmic CTLA-4 currently remains unknown.

\section{Immune checkpoint inhibitor-related hypophysitis in the pediatric population}

The first study that documented ICI use in children was in 2016, where 33 patients treated with ipilimumab were enrolled in a phase I trial. Numerous advanced solid tumors were the target of ICI therapy, with unresectable stage IIIc or stage IV melanoma being the most common. ${ }^{44}$ Serial monitoring of pituitary function was performed during the trial, and one patient was found to have ICl-related hypophysitis after the development of severe headaches, vision changes, and diabetes insipidus. Following treatment with glucocorticoid replacement, the patient's symptoms improved, but panhypopituitarism persisted, requiring continued full hormone replacement therapies. In March 2017, the FDA approved pembrolizumab for the treatment of both adults and children with refractory classic Hodgkin lymphoma or those who relapsed after three or more prior treatments. ${ }^{45}$

In three early-phase clinical trials published in 2020, which included single-agent ICI therapy with PD-1 (pembrolizumab and nivolumab) and PD-L1 inhibitors (atezolizumab), no reports of hypophysitis were observed in a combined total of 251 patients. ${ }^{4--48}$ However, there was one case of adrenal insufficiency in a patient treated with pembrolizumab, but it was unclear if this patient developed primary or secondary adrenal insufficiency. ${ }^{47}$ It is possible that the low prevalence of ICl-related hypophysitis in pediatric patients may be due to the small number of patients being treated compared with adults, and a lack of formal assessment of overall pituitary function because of mild non-specific symptoms, or that pediatric patients may not report any symptoms. As the use of $\mathrm{ICI}$ therapy expands in the pediatric population in the coming years, it is conceivable that the incidence of reported side-effects will increase and therefore, it is important that pediatricians are aware of ICl-related hypophysitis, and undertake baseline and periodic endocrine assessments of all pediatric patients undergoing $\mathrm{ICl}$ therapy and after treatment completion.

\section{Clinical manifestations of immune checkpoint inhibitor-related hypophysitis}

Hypophysitis can present at any time point during or after ICI therapy. A study of 129 patients treated with ipilimumab for malignant melanoma demonstrated that hypophysitis tended to occur later in the treatment course, with the mean onset being 63 days post-treatment initiation (range 41-119 days). There were also documented ICl-related hypophysitis cases after completion of the standard 9-week course that contrasts to ICI-related side effects of other organs such as thyroid, gastrointestinal tract, and lungs, which are more prone to develop while being on treatment. ${ }^{14}$

The clinical manifestation of hypophysitis varies, ranging from being clinically asymptomatic, to having one or more features of pituitary hormone deficits, to the acute onset of mass-effect symptoms (e.g., headache with or without nausea, and visual disturbances that include visual field deficits and ophthalmoplegia). ${ }^{49}$ On the other hand, the onset of headache and visual symptoms may be insidious, subacute, or acute, and even mimic symptoms of pituitary apoplexy. Because these symptoms can be vague, 


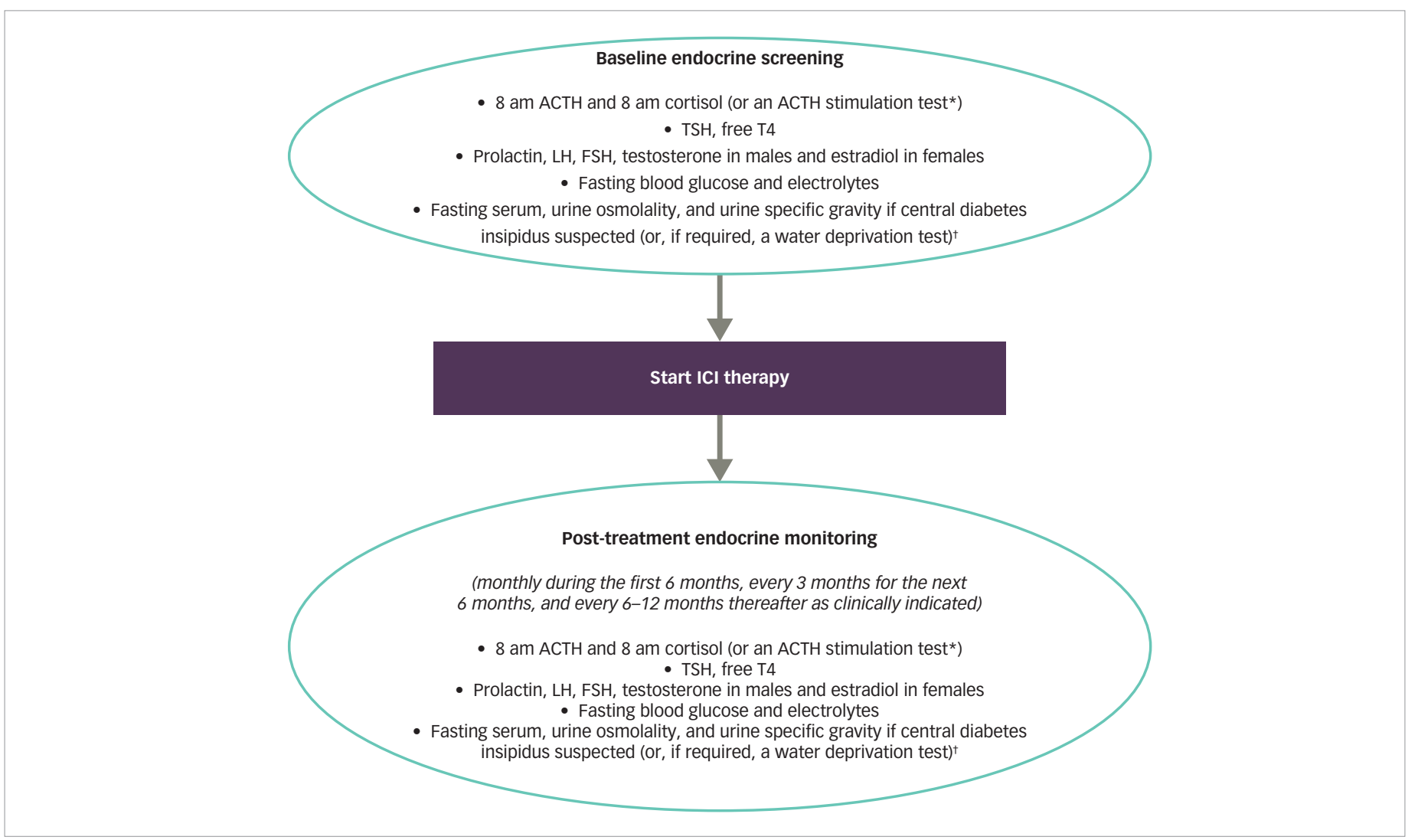

*Baseline and/or post ICl-therapy ACTH stimulation test may be considered in cases of equivocal 8 am serum cortisol levels. Caution must be used in interpreting the results of post ICl-therapy ACTH stimulation test, as it may be falsely normal in the setting of acute ACTH and cortisol deficiencies.

${ }^{\dagger}$ May be performed at the clinician's discretion depending on if the patient reports symptoms of excessive thirst, polydipsia, and hypotonic polyuria.

ACTH = adrenocorticotropic hormone; FSH = follicle-stimulating hormone; ICl = immune checkpoint inhibitor; $\mathrm{LH}=$ luteinizing hormone; $T S H=$ thyroid-stimulating hormone.

they may go unnoticed by physicians unfamiliar with these medications and clinical presentation. It is, however, noteworthy that the severity of pituitary hormonal deficits can be variable and may be disproportionate to the magnetic resonance imaging (MRI) findings. ${ }^{50,51}$ Furthermore, there is no predictable order in which anterior pituitary hormone deficits develops. ${ }^{50,51}$

The most serious presentation is related to secondary adrenal insufficiency, which in severe cases, can result in life-threatening adrenal crisis, especially if not promptly recognized and treated accordingly. Although there are no published reports in the literature of adrenal crisis occurring in patients with ICl-related hypophysitis, it is well-known that cancer patients are highly susceptible to infections ${ }^{52-54}$ and gastrointestinal disorders; 55,56 two common precipitants to the development of an adrenal crisis. Therefore, the risk of developing adrenal crisis is still inherently present; hence, it is very important that clinicians educate patients about this potential complication, and the ability to stress dose, including self-administration of parenteral glucocorticoids. In less severe forms of adrenal insufficiency, patients may present with non-specific symptoms of fatigue or nausea, both of which are also relatively common symptoms in patients with cancer. ${ }^{57}$ Similarly, symptoms of secondary hypothyroidism may range from being mild to severe life-threatening myxedema. Patients with secondary hypogonadism may experience symptoms commonly associated with hypogonadism, such as menstrual abnormalities, decreased libido and impaired sexual function. Those with central diabetes insipidus may present with excessive thirst, polydipsia, and hypotonic polyuria. However, in contrast to lymphocytic hypophysitis, visual disturbances from pituitary enlargement and optic chiasm compression are relatively less common in patients with ICl-related hypophysitis.

\section{Diagnosis of immune checkpoint inhibitor-related hypophysitis}

Prior to the initiation of $\mathrm{ICl}$ therapy, baseline measurements of basal pituitary hormones (8 am ACTH, 8 am cortisol, TSH, free T4, FSH, LH, prolactin, and testosterone in males or estradiol in premenopausal females), fasting glucose, and electrolytes should be obtained (Figure 1). Depending on whether the patient reports excessive thirst, polydipsia, and hypotonic polyuria that raises the possibility of central diabetes insipidus, fasting serum, urine osmolality, and urine specific gravity may be performed at the clinician's discretion, and if the diagnosis remains equivocal, a water deprivation test may be considered (Figure 2).

Hypoglycemia, hyponatremia, and hyperkalemia suggest adrenal insufficiency, but are not specific, and therefore cannot be used alone for diagnosis. Furthermore, hyponatremia with normokalemia may be present in secondary adrenal insufficiency due to excess vasopressin secretion, ${ }^{58}$ but hyperkalemia would not be an expected finding as adrenal mineralocorticoid secretion is controlled primarily through the reninangiotensin-aldosterone system, which would be intact in these cases. ${ }^{59}$ 


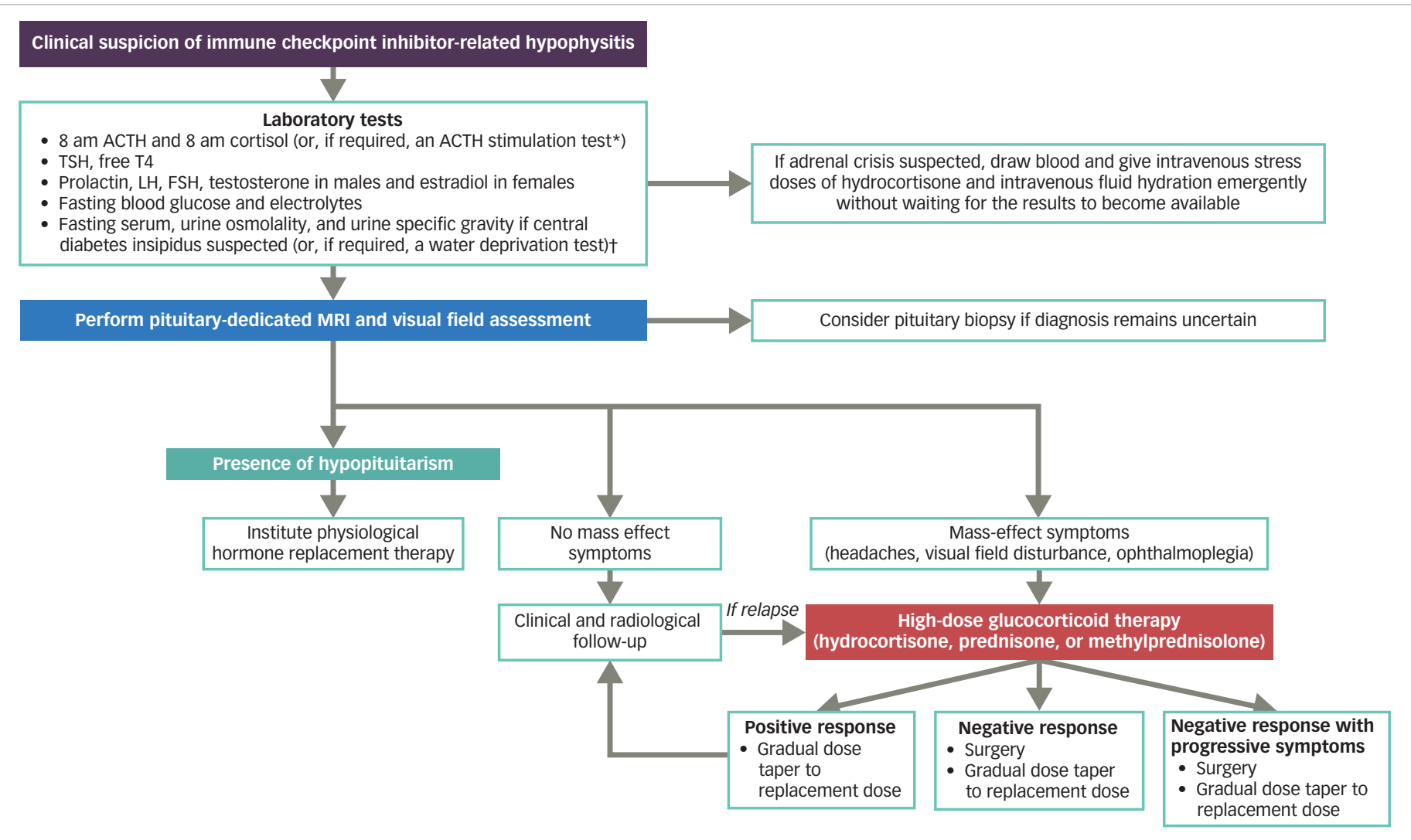

*Baseline and/or post ICl-therapy ACTH stimulation test may be considered in cases of equivocal 8 am serum cortisol levels. Caution must be used in interpreting the results of post ICl-therapy ACTH stimulation test, as it may be falsely normal in the setting of acute ACTH and cortisol deficiencies.

${ }^{\dagger}$ May be performed at the clinician's discretion depending on whether the patient reports symptoms of excessive thirst, polydipsia, and hypotonic polyuria.

$\mathrm{ACTH}=$ adrenocorticotropic hormone; $\mathrm{FSH}=$ follicle-stimulating hormone; ICl = immune checkpoint inhibitor; $\mathrm{LH}=\mathrm{luteinizing}$ hormone; $\mathrm{MRI}=\mathrm{magnetic}$ resonance imaging;

TSH = thyroid-stimulating hormone.

However, if hyponatremia, hyperkalemia, and elevated serum ACTH and renin levels in the setting of low serum cortisol levels are found, then the possibility of primary adrenal insufficiency caused by autoimmune adrenalitis should be entertained, albeit rare in the immunotherapy context. ${ }^{60}$ Conversely, hyponatremia and hypokalemia may occur in patients with cancer as a result of chronic diarrhea and vomiting from the effects of chemotherapy. Therefore, in the appropriate clinical context and especially in the presence of hyponatremia and hypotension, am serum cortisol levels $<3 \mathrm{ug} / \mathrm{dL}$ are highly suggestive, whereas levels $>15 \mathrm{ug} / \mathrm{dL}$ are unlikely to indicate adrenal insufficiency. ${ }^{59,61,62}$ Values in between 3 and $15 \mathrm{ug} / \mathrm{dL}$ are equivocal, and in this context, ACTH stimulation testing may be considered, 59,61,62 although the result should be interpreted with some degree of caution as it may be falsely normal in the setting of acute hypophysitis, because the adrenal glands have not yet atrophied and may still respond to exogenous ACTH stimulation..$^{63}$ By contrast, failure to respond to ACTH stimulation to a serum cortisol level of $\geq 18 \mathrm{ug} / \mathrm{dL}$ suggests adrenal insufficiency. ${ }^{59}$

After $\mathrm{ICl}$ therapy has been initiated, we recommend periodic endocrine monitoring during the first 6 months, every 3 months for the next 6 months, and every 6-12 months thereafter as clinically indicated (Figure 1).61,62 It is noteworthy that non-stimulated 8 am ACTH levels may be helpful to differentiate between primary and secondary adrenal insufficiency (as both are possible consequences of $\mathrm{ICl}$ therapy) because treatment for primary adrenal insufficiency requires the inclusion of mineralocorticoid replacement in addition to glucocorticoid replacement, whereas secondary adrenal insufficiency requires only glucocorticoid replacement. ${ }^{59}$

If secondary adrenal insufficiency is confirmed, it is important to note that recovery of pituitary corticotroph function is less likely, and patients will need lifelong glucocorticoid replacement therapy; ${ }^{32}$ unlike the thyrotroph and gonadotroph axes, where function may be regained over time. Similarly, distinction should be made between primary and secondary hypothyroidism and hypogonadism. Serum TSH and gonadotropin levels for secondary hypothyroidism and hypogonadism may be low or inappropriately normal, and thus, serum free T4, testosterone, and estradiol levels should be obtained. ${ }^{59}$ The presence of diabetes insipidus should prompt consideration of a metastatic lesion to the pituitary gland, as diabetes insipidus is more common with metastases than ICl-related hypophysitis. Conversely, measurement of serum IGF-I levels is not required, because even if growth hormone deficiency is suspected, growth hormone replacement is contraindicated due to the oncological context. ${ }^{64}$

Imaging with a pituitary-dedicated MRI with and without contrast is recommended if patients report headache and/or visual disturbance. In ICl-related hypophysitis, MRI typically shows diffuse enlargement and/or enhancement of the pituitary gland, although up to $25 \%$ of scans may appear normal and therefore should not be used to rule out hypophysitis. ${ }^{65}$ 
MRI can also help to rule out other potential pituitary lesions such as metastatic disease and apoplexy. However, there is no consensus on how often follow-up MRIs should be obtained once ICl-related hypophysitis is confirmed. Therefore, in order not to miss the diagnosis, it is important that clinicians be alert to signs and symptoms of ICl-related hypophysitis, and to continue periodic screening for endocrine dysfunction, even after completion of immunotherapy (Figure 1).

\section{Grading system of adverse events of immune checkpoint inhibitors}

The National Cancer Institute has recommended that AEs in patients with cancer treated with chemotherapy be graded as per the Common Terminology Criteria for Adverse Events according to severity. ${ }^{66}$ Grades range from 1-5: grade 1 is defined as asymptomatic or mild symptoms not needing intervention; grade 2 is defined as moderate, impacting age-appropriate activities of daily living and requiring minimal intervention; grade 3 is severe, not life-threatening but impacting activities of daily living; grade 4 is a life-threatening situation requiring urgent intervention; and grade 5 is death. Once the diagnosis of $\mathrm{ICl}$ hypophysitis is made, determination of irAE grade is the next step. There is currently no consensus among various society guidelines as to treatment for ICl-related hypophysitis based on irAE grading, although in general, treatment recommendations follow a similar pattern to non-ICl-related hypophysitis. ${ }^{67,68}$

\section{Treatment}

The primary goals of treatment of ICl-related hypophysitis are to replace any underlying hormonal deficits and to provide any relief of neurological and mass-effect symptoms. However, currently, there are no consensus guidelines on the optimal treatment of ICI-related hypophysitis because of the rarity of the disease, heterogeneity in clinical presentation, and lack of clinical trials comparing the efficacy of different treatment modalities. ${ }^{35}$

An important point to clarify is the use of glucocorticoids for ICl-related irAEs, as they are used for two different purposes. The primary and most common use is as an immunosuppressant aimed at counteracting the immune-system activation intentionally caused by $\mathrm{ICl}$ therapy. ${ }^{61-63}$ The endocrine system, however, is somewhat unique in that glucocorticoids are used both for their immune-modulating properties as well as for replacement of cortisol deficiency resulting from $\mathrm{ICl}$-induced primary or secondary adrenal insufficiency. During the acute phase of hypophysitis, the development of an adrenal crisis and the presence of mass effects are strong indicators for emergent treatment. High-dose glucocorticoid therapy is generally utilized to reduce the mass effect, but there is little evidence to suggest its direct role in promoting recovery of pituitary function, ${ }^{69}$ and conversely, may even reduce survival rates in patients with melanoma.70 When patients present with acute hypophysitis, thorough assessment of pituitary function should be conducted, and hormone replacement management instituted for any endocrinopathies detected. Surgery is generally not required, but can be considered if the mass effect persists despite high-dose glucocorticoid therapy, or if tissue diagnosis is required to confirm the pathology of the sellar lesion. Disadvantages of surgery include recurrence of the lesion and the development of post-operative hypopituitarism. In cases without mass effect, treatment with hormone replacement therapy for any pituitary hormone deficits and close monitoring are all that is needed, as spontaneous resolution of pituitary enlargement over time has been reported in some cases. ${ }^{71,72}$
Glucocorticoid therapy to utilize its immune-modulating effects can be considered in irAE grade 2, although literature supporting its use in hypophysitis is currently lacking. ${ }^{69}$ Recommendations for irAE grades 3 and 4 suggest discontinuation of $\mathrm{ICI}$ therapy, hormone replacement as indicated, and consideration of high-dose glucocorticoid therapy. ${ }^{73}$ For secondary adrenal insufficiency, patients with irAEs grades 1 and 2 can generally be managed with physiologic glucocorticoid replacement doses of hydrocortisone (15-25 mg in divided daily doses) or prednisone (5.0-7.5 mg a day), ${ }^{59}$ education on sick day rules and appropriate stress dosing, and continuation of ICl therapy. Medic Alert card/necklace/bracelet and adequate refills of injectable hydrocortisone or dexamethasone with instructions about its handling should also be implemented. ${ }^{59}$ In some cases of grade 3 hypophysitis without adrenal crisis or mass-effect symptoms, it may be possible to avoid high-dose glucocorticoid therapy and utilize physiologic replacement doses. ${ }^{74}$ If, however, the patient has secondary adrenal insufficiency and is symptomatic requiring hospitalization, stress doses of glucocorticoids should be administered (Figure 2). ${ }^{61-63}$

In the case of a life-threatening illness and suspected adrenal crisis without mass-effect symptoms, empiric high-dose glucocorticoid therapy should be administered immediately (e.g., intravenous or intramuscular bolus injection of $100 \mathrm{mg}$ hydrocortisone followed by continuous intravenous infusion of $200 \mathrm{mg}$ hydrocortisone over 24 hours or $50 \mathrm{mg}$ intravenous or intramuscular hydrocortisone injections every 6 hours) (Figure 2). ${ }^{63,75,76}$ If mass-effect symptoms are present, high-dose glucocorticoid therapy (e.g., intravenous or intramuscular bolus injection of $100 \mathrm{mg}$ hydrocortisone followed by continuous intravenous infusion of $200 \mathrm{mg}$ hydrocortisone over 24 hours or $50 \mathrm{mg}$ intravenous or intramuscular hydrocortisone injections every 6 hours or $1-2 \mathrm{mg} / \mathrm{kg} /$ day of prednisone or equivalent) should be emergently administered and $\mathrm{ICl}$ therapy discontinued.61-63 Methylprednisolone should not be used as first-line treatment for acute adrenal insufficiency and adrenal crisis due to its prolonged duration of action compared with hydrocortisone and prednisone, but may be used for treatment of mass-effect symptoms because of its potent anti-inflammatory action. ${ }^{77}$ If a positive response is observed, perform gradual glucocorticoid dose taper to replacement doses and re-introduce $\mathrm{ICI}$ therapy. In the event of a negative response with or without progressive visual symptoms, consider surgery to relieve mass effect and gradually perform glucocorticoid dose taper to replacement doses and consider re-introducing $\mathrm{ICI}$ therapy, although the timeline remains unclear (Figure 2) ${ }^{78}$

If mass-effect symptoms are present without secondary adrenal insufficiency, high-dose hydrocortisone, prednisone, or methylprednisolone may still be used until there is documented MRI and clinical evidence of improvement of mass-effect symptoms, allowing gradual glucocorticoid dose taper can then be commenced. ${ }^{79}$ Because approximately one-third of patients receiving $\mathrm{ICl}$ therapy will require high-dose glucocorticoid therapy for management of irAEs, ${ }^{73}$ these patients are also susceptible to adrenal suppression, hyperglycemia, and osteoporosis. Thus, it is possible that some patients with ICl-related hypophysitis may develop high-dose glucocorticoid-induced adrenal suppression, and differentiating these patients from those with ICl-induced secondary adrenal insufficiency is important but may be challenging when the high doses of glucocorticoids require dose tapering and discontinuation. Therefore, long-term monitoring of the hypothalamic-pituitary-adrenal axis is imperative, even after completion of $\mathrm{ICl}$ therapy, as the axis may recover in patients with high-dose glucocorticoid-induced adrenal suppression, whereas those with secondary adrenal insufficiency may not. 
For secondary hypothyroidism, levothyroxine therapy (average dose of $1.6 \mathrm{\mu g} / \mathrm{kg} /$ day) is recommended, and measurements of free thyroxine levels, conducted at 6-12 week intervals initially, with the goal of achieving levels between mid to upper half of the reference range. ${ }^{59}$ If both adrenal insufficiency and hypothyroidism are confirmed in the non-acute setting, glucocorticoid followed by thyroid hormone replacement should be initiated in that order prior to $\mathrm{ICl}$ therapy initiation, because without glucocorticoid replacement, thyroid hormone replacement may unmask or exacerbate adrenal insufficiency and precipitate an adrenal crisis due to its effects in increasing cortisol clearance. For secondary hypogonadism, testosterone replacement in males may be considered, and the choice of the testosterone formulation depends on the patient preference and insurance coverage, whereas hormone replacement therapy in pre-menopausal females should be provided (e.g., estrogen-progestin formulations for females with intact uterus and estrogen-only formulations for females who have previously undergone a hysterectomy). ${ }^{59}$

For hyperprolactinemia, often no treatment is necessary for males, whereas treatment with dopamine agonists may be considered in symptomatic females with bothersome galactorrhea and menstrual abnormalities, with dose adjustments to normalize serum prolactin levels. ${ }^{80}$ For central diabetes insipidus, treatment with oral or intra-nasal desmopressin can be initiated in symptomatic patients, with careful monitoring of fluid intake and urine output, with adjusted desmopressin dosing based on urine output and serum sodium levels. ${ }^{59}$ It is important to be aware that secondary hypothyroidism and secondary hypogonadism from $\mathrm{ICl}$ may be transient, and pituitary function for these hormonal axes may recover spontaneously over time requiring discontinuation of their respective hormone replacement therapies, whereas ICl-related secondary adrenal insufficiency tends to persist in most cases.?

\section{Summary}

$\mathrm{ICl}$ therapy is a promising new agent in the oncologic realm, and its use is increasing both in adults and, more recently, in children. While it has proven to be effective in treating several different cancers, unwanted immune system activation against host tissue is an unfortunate reality. Knowledge of ICls and their potential side effects, as well as proper surveillance, will hopefully lead to earlier diagnosis and treatment. It is imperative that clinicians are mindful of this class of medications and their potential side effects. Hypophysitis should be considered in patients on $\mathrm{ICl}$ therapy, particularly CTLA-4 inhibitors, such as ipilimumab; or those who have clinical or biochemical evidence of adrenal insufficiency, hypothyroidism, hypogonadism, hyperprolactinemia, or diabetes insipidus with or without headache or vision changes. Prompt recognition of this condition, along with proper treatment, may result in some recovery of pituitary function, although this is unlikely if secondary adrenal insufficiency is present and patients need to be counseled accordingly. As the indications and the number of patients treated with ICIs continue to expand, the number of irAE episodes will inevitably increase, and appropriate management of these events should be conducted collaboratively between the treating oncologist and endocrinologist. $\square$
1. Kelly PN. The cancer immunotherapy revolution. Science. 2018;359:1344-5

2. Ribas A, Wolchok JD. Cancer immunotherapy using checkpoin blockade. Science. 2018;359:1350-5.

3. Pardoll DM. The blockade of immune checkpoints in cancer immunotherapy. Nat Rev Cancer. 2012;12:252-64.

4. Leach DR, Krummel MF, Allison JP. Enhancement of antitumor immunity by CTLA-4 blockade. Science. 1996;271:1734-6.

5. Emens LA, Ascierto PA, Darcy PK, et al. Cancer immunotherapy: opportunities and challenges in the rapidly evolving clinical landscape. Eur J Cancer. 2017;81:116-29.

6. Herrscher $\mathrm{H}$, Robert C. Immune checkpoint inhibitors in melanoma in the metastatic, neoadjuvant, and adjuvant setting. Curr Opin Oncol. 2020;32:106-13.

7. Chang LS, Barroso-Sousa R, Tolaney SM, et al. Endocrine toxicity of cancer immunotherapy targeting immune checkpoints. Endocr Rev. 2019;40:17-65.

8. Kumar BV, Connors TJ, Farber DL. Human T cell development, Iocalization, and function throughout life. Immunity. 2018;48:202-13.

9. Naidoo J, Page DB, Wolchok JD. Immune checkpoint blockade Hematol Oncol Clin North Am. 2014;28:585-600.

10. Granier $C$, De Guillebon $E$, Blanc $C$, et al. Mechanisms of action and rationale for the use of checkpoint inhibitors in cancer. ESMO Open. 2017;2:e000213.

11. Littman DR. Releasing the brakes on cancer immunotherapy. Cell. 2015;162:1186-90.

12. Topalian SL, Drake CG, Pardoll DM. Immune checkpoint blockade: a common denominator approach to cancer therapy. Cancer Cell. 2015;27:450-61.

13. Gatalica Z, Snyder C, Maney T, et al. Programmed cell death 1 (PD-1) and its ligand (PD-L1) in common cancers and their correlation with molecular cancer type. Cancer Epidemiol Biomarkers Prev. 2014;23:2965-70.

14. Hodi FS, O'Day SJ, MCDermott DF, et al. Improved survival with ipilimumab in patients with metastatic melanoma. N Eng/ J Med. 2010;363:711-23.

15. O'Day SJ, Maio M, Chiarion-Sileni V, et al. Efficacy and safety of ipilimumab monotherapy in patients with pretreated advanced melanoma: a multicenter single-arm phase II study. Ann Oncol. 2010:21:1712-7.

16. Garon EB, Rizvi NA, Hui R, et al. Pembrolizumab for the treatment of non-small-cell lung cancer. N Engl J Med. 2015;372:2018-28.

17. Homet Moreno B, Ribas A. Anti-programmed cell death protein-1/ligand-1 therapy in different cancers. Br J Cancer. 2015;112:1421-7.

18. Shitara K, Ozguroglu M, Bang YJ, et al. Pembrolizumab versus paclitaxel for previously treated, advanced gastric or gastro-oesophageal junction cancer (KEYNOTE-061): a randomised, open-label, controlled, phase 3 trial. Lancet.
2018;392:123-33.

9. Hodi FS, Chiarion-Sileni V, Gonzalez R, et al. Nivolumab plus ipilimumab or nivolumab alone versus ipilimumab alone in advanced melanoma (CheckMate 067): 4-year outcomes of a multicentre, randomised, phase 3 trial. Lancet Oncol. 2018;19:1480-92

20. Krishnamurthy A, Jimeno A. Atezolizumab: A novel PD-L1 inhibito in cancer therapy with a focus in bladder and non-small cell lung cancers. Drugs Today (Barc). 2017:53:217-37.

21. Powles T, O'Donnell PH, Massard C, et al. Efficacy and safety of durvalumab in locally advanced or metastatic urothelial carcinoma: updated results from a phase 1/2 open-label study. JAMA Oncol. 2017;3:e172411.

22. Teets A, Pham L, Tran EL, et al. Avelumab: a novel anti-PD-L1 agent in the treatment of Merkel cell carcinoma and urothelial cell carcinoma. Crit Rev Immunol. 2018:38:159-206.

23. Migden MR, Rischin D, Schmults CD, et al. PD-1 blockade with cemiplimab in advanced cutaneous squamous cell carcinoma. N Eng/ J Med. 2018;379:341-51

24. De velasco G, Je Y, Bosse D, et al. Comprehensive meta-analysis of key immune-related adverse events from CTLA-4 and PD-1/PD-L inhibitors in cancer patients. Cancer Immunol Res. 2017;5:312-8.

25. Hansen ED, Wang X, Case AA, et al. Immune checkpoint inhibitor toxicity review for the palliative care clinician. J Pain Symptom Manage. 2018:56:460-72.

26. Puzanov I, Diab A, Abdallah K, et al; Society for Immunotherapy of Cancer Toxicity Management Working Group. Managing toxicities associated with immune checkpoint inhibitors: consensus recommendations from the Society for Immunotherapy of Cancer (SITC) Toxicity Management Working Group. J Immunother Cancer. 2017:5:95.

27. Piranavan P, Li Y, Brown E, et al. Immune checkpoint inhibitor-induced hypoparathyroidism associated with calcium-sensing receptor-activating autoantibodies. I Clin Endocrinol Metab. 2019;104:550-6.

28. Raschi E, Mazzarella A, Antonazzo IC, et al. Toxicities with immun checkpoint inhibitors: emerging priorities from disproportionality analysis of the FDA adverse event reporting system. Target Onco. 2019;14:205-21.

29. Trinh B, Sanchez GO, Herzig P, Laubli H. Inflammation-induced hypoparathyroidism triggered by combination immune checkpoint blockade for melanoma. J Immunother Cancer. 2019;7:52

30. de Filette J, Andreescu CE, Cools F, et al. A systematic review and meta-analysis of endocrine-related adverse events associated with immune checkpoint inhibitors. Horm Metab Res. 2019;51:145-56.

31. Antonelli A, Ferrari SM, Fallahi P. Current and future immunotherapies for thyroid cancer. Expert Rev Anticancer Ther. 2018;18:149-59.
32. Kassi E, Angelousi A, Asonitis $\mathrm{N}$, et al. Endocrine-related adverse events associated with immune-checkpoint inhibitors in patients with melanoma. Cancer Med. 2019;8:6585-94.

33. Albarel F, Gaudy C, Castinetti F, et al. Long-term follow-up of ipilimumab-induced hypophysitis, a common adverse event of the anti-CTLA-4 antibody in melanoma. Eur J Endocrinol. 2015;172:195-204.

34. Deligiorgi MV, Siasos G, Vergadis C, Trafalis DT. Centra diabetes insipidus related to anti-programmed cell-death 1 protein active immunotherapy. Int Immunopharmacol. 2020;83:106427

35. Guaraldi F, Giordano R, Grottoli S, et al. Pituitary autoimmunity. Front Horm Res. 2017;48:48-68

36. Javanbakht A, D'Apuzzo M, Badie B, Salehian B. Pituitary metastasis: a rare condition. Endocr Connect. 2018;7:1049-57.

37. Kanie K, Iguchi G, Bando H, et al. Two cases of atezolizumab-induced hypophysitis. J Endocr Soc. 2018;2:91-5.

38. Lupi I, Brancatella A, Cosottini M, et al. Clinical heterogeneity of hypophysitis secondary to PD-1/PD-L1 blockade: insights from four cases. Endocrinol Diabetes Metab Case Rep. 2019;2019:19-0102.

39. Attia P, Phan GQ, Maker AV, et al. Autoimmunity correlates with tumor regression in patients with metastatic melanoma treated with anti-cytotoxic T-lymphocyte antigen-4. J Clin Oncol. 2005;23:6043-53

40. Faje AT, Sullivan R, Lawrence D, et al. Ipilimumab-induced hypophysitis: a detailed longitudinal analysis in a large cohort of patients with metastatic melanoma. J Clin Endocrinol Metab. 2014;99:4078-85.

41. Maker AV, Yang JC, Sherry RM, et al. Intrapatient dose escalation of anti-CTLA-4 antibody in patients with metastatic melanoma. J Immunother. 2006:29:455-63.

42. Gubbi S, Hannah-Shmouni F, Verbalis JG, Koch CA. Hypophysitis: an update on the novel forms, diagnosis and management of disorders of pituitary inflammation. Best Pract Res Clin Endocrinol Metab. 2019;33:101371.

43. Caturegli P, Di Dalmazi G, Lombardi M, et al. Hypophysitis secondary to cytotoxic T-lymphocyte-associated protein 4 blockade: insights into pathogenesis from an autopsy series Am J Pathol. 2016;186:3225-35.

44. Merchant MS, Wright M, Baird K, et al. Phase I clinical trial of ipilimumab in pediatric patients with advanced solid tumors. Clin Cancer Res. 2016;22:1364-70

45. Chen R, Zinzani PL, Fanale MA, et al. Phase II study of the efficacy and safety of pembrolizumab for relapsed/refractory classic Hodgkin lymphoma. J Clin Oncol. 2017;35:2125-32.

46. Davis KL, FoX E, Merchant MS, et al. Nivolumab in children and young adults with relapsed or refractory solid tumours or lymphoma (ADVL1412): a multicentre, open-label, single-arm, phase 1-2 trial. Lancet Oncol. 2020;21:541-50. 
47. Geoerger B, Kang HJ, Yalon-Oren M, et al. Pembrolizumab in paediatric patients with advanced melanoma or a PD-L1-positive, advanced, relapsed, or refractory solid tumour or lymphoma (KEYNOTE-051): interim analysis of an open-label, single-arm, phase 1-2 trial. Lancet Oncol. 2020;21:121-33.

48. Geoerger B, Zwaan CM, Marshall LV, et al. Atezolizumab for children and young adults with previously treated solid tumours, non-Hodgkin lymphoma, and Hodgkin lymphoma (iMATRIX): a multicentre phase 1-2 study. Lancet Oncol. 2020;21:134-44.

49. Yuen KCJ, Moloney KJ, Mercado JU, et al. A case series of atypica features of patients with biopsy-proven isolated IgG4-related hypophysitis and normal serum IgG4 levels. Pituitary. 2018;21:238-46.

50. Caturegli P, Lupi I, Landek-Salgado M, et al. Pituitary autoimmunity: 30 years later. Autoimmun Rev. 2008;7:631-7.

51. Caturegli $P$, Newschaffer $C$, Olivi $A$, et al. Autoimmune hypophysitis. Endocr Rev. 2005;26:599-614

52. Bodro M, Gudiol C, Garcia-Vidal C, et al. Epidemiology, antibiotic therapy and outcomes of bacteremia caused by drug-resistant ESKAPE pathogens in cancer patients. Support Care Cancer. 2014;22:603-10.

53. Lalaoui $R$, Javelle $E$, Bakour $S$, et al. Infections due to carbapenem-resistant bacteria in patients with hematologic malignancies. Front Microbiol. 2020;11:1422.

54. Wisplinghoff $\mathrm{H}$, Seifert $\mathrm{H}$, Wenzel RP, Edmond MB. Current trends in the epidemiology of nosocomial bloodstream infections in patients with hematological malignancies and solid neoplasms in hospitals in the United States. Clin Infect Dis. 2003;36:1103-10.

55. Naeim A, Dy SM, Lorenz KA, et al. Evidence-based recommendations for cancer nausea and vomiting J I Clin Oncol. 2008;26:3903-10.

56. Stephenson J, Davies A. An assessment of aetiology-based guidelines for the management of nausea and vomiting in patients with advanced cancer. Support Care Cancer. 2006;14:348-53

57. Postow MA. Managing immune checkpoint-blocking antibody side effects. Am Soc Clin Oncol Educ Book. 2015;76-83.

58. Oelkers W. Hyponatremia and inappropriate secretion of vasopressin (antidiuretic hormone) in patients with hypopituitarism. N Eng/ J Med. 1989;321:492-6.

59. Fleseriu M, Hashim IA, Karavitaki N, et al. Hormonal replacement in hypopituitarism in adults: an Endocrine Society Clinical Practice Guideline. I Clin Endocrinol Metab. 2016;101:3888-921

60. Grouthier V, Lebrun-Vignes B, Moey M, et al. Immune checkpoint inhibitor-associated primary adrenal insufficiency: WHO VigiBase report analysis. Oncologist. 2020;10:696-701

61. Del Rivero J, Cordes LM, Klubo-Gwiezdzinska J, et al. Endocrine-related adverse events related to immune checkpoint inhibitors: proposed algorithms for management. Oncologist. 2020;25:290-300

62. Ferrari SM, Fallahi P, Elia G, et al. Autoimmune endocrine dysfunctions associated with cancer immunotherapies. Int Mol Sci. 2019;20:2560.

63. Higham CE, Olsson-Brown A, Carroll P, et al; Society fo Endocrinology Clinical Committee. Society for Endocrinology Endocrine Emergency Guidance: Acute management of the endocrine complications of checkpoint inhibitor therapy. Endocr Connect. 2018;7:G1-7.

64. Castinetti F, Albarel F, Archambeaud F, et al. Endocrine side-effects of new anticancer therapies: overall monitoring and conclusions. Ann Endocrinol (Paris). 2018;79:591-5.

65. Ryder M, Callahan M, Postow MA, et al. Endocrine-related adverse events following ipilimumab in patients with advanced melanoma: a comprehensive retrospective review from a single institution. Endocr Relat Cancer. 2014;21:371-81

66. National Cancer Institute, Common Terminology Criteria for Adverse Events (CTCAE), 2017. Available at: https://ctep.cancer. gov/protocolDevelopment/electronic applications/ctc.htm (accessed October 29, 2020).

67. Angelousi A, Alexandraki KI, Tsoli M, et al. Hypophysitis (including IgG4 and immunotherapy). Neuroendocrinology. 2020;110:822-35.

68. Lojou M, Bonneville JF, Ebbo M, et al. IgG4 hypophysitis: Diagnosis and management. Presse Med. 2020;49:104016.

69. Min L, Hodi FS, Giobbie-Hurder A, et al. Systemic high-dose corticosteroid treatment does not improve the outcome of ipilimumab-related hypophysitis: a retrospective cohort study.
Clin Cancer Res. 2015;21:749-55.

70. Faje AT, Lawrence D, Flaherty K, et al. High-dose glucocorticoids for the treatment of ipilimumab-induced hypophysitis is associated with reduced survival in patients with melanoma. Cancer. 2018:124:3706-14.

71. Honegger J, Buchfelder M, Schlaffer S, et al, Pituitary Working Group of the German Society of Endocrinology. Treatment of primary hypophysitis in Germany. I Clin Endocrinol Metab. 2015;100:3460-9.

72. Khare S, Jagtap VS, Budyal SR, et al. Primary (autoimmune) hypophysitis: a single centre experience. Pituitary. 2015;18:16-22.

73. Horvat TZ, Adel NG, Dang TO, et al. Immune-related adverse events, need for systemic immunosuppression, and effects on survival and time to treatment failure in patients with melanoma treated with ipilimumab at Memorial Sloan Kettering Cancer Center. J Clin Oncol. Memorial Sloan Kett

74. Castinetti F, Albarel F, Archambeaud F, et al. French Endocrine Society guidance on endocrine side effects of immunotherapy. Endocr Relat Cancer. 2019;26:G1-18.

75. Arlt W, Baldeweg SE, Pearce SHS, Simpson HL. Endocrinology in the time of COVID-19: Management of adrenal insufficiency. Eur I Endocrinol. 2020;183:G25-32.

76. Prete $A$, Taylor $A E$, Bancos I, et al. Prevention of adrenal crisis: cortisol responses to major stress compared to stress dose hydrocortisone delivery. 1 Clin Endocrinol Metab. 2020;105:2262-74

77. Williams DM. Clinical pharmacology of corticosteroids. Respir Care. 2018;63:655-70.

78. Haanen J, Carbonnel F, Robert C, et al. Management of toxicities from immunotherapy: ESMO Clinical Practice Guidelines for diagnosis, treatment and follow-up. Ann Oncol. 2017;28(Suppl. 4):iv119-42.

79. Weber JS, Kahler KC, Hauschild A. Management of immune-related adverse events and kinetics of response with ipilimumab. J Clin Oncol. 2012;30:2691-7.

80. Melmed S, Casanueva FF, Hoffman AR, et al; Endocrine Society. Diagnosis and treatment of hyperprolactinemia: an Endocrine Society clinical practice guideline. J Clin Endocrinol Metab. 2011;96:273-88. 